\title{
Vigna Barberini (Palatin, Rome)
}

Campagne d'études 2015

\section{Françoise Villedieu}

\section{OpenEdition}

\section{Journals}

Édition électronique

URL : http://journals.openedition.org/cefr/1485

DOI : $10.4000 /$ cefr. 1485

ISSN : 2282-5703

Éditeur

École française de Rome

Référence électronique

Françoise Villedieu, «Vigna Barberini (Palatin, Rome) 》, Chronique des activités archéologiques de l'École française de Rome [En ligne], Italie centrale, mis en ligne le 25 février 2016, consulté le 15 novembre 2019. URL : http://journals.openedition.org/cefr/1485; DOI : 10.4000/cefr.1485

Ce document a été généré automatiquement le 15 novembre 2019.

(c) École française de Rome 


\title{
Vigna Barberini (Palatin, Rome)
}

\author{
Campagne d'études 2015 \\ Françoise Villedieu
}

1 À l'issue de trois campagnes de fouille menées sur le terrain et en l'absence de perspectives favorables à la poursuite de ces travaux, en 2015, nous avons pris le parti de publier les données recueillies. Ces dernières ont été livrées par les recherches effectuées en 2009, en 2010 et en 2014 ${ }^{1}$. L'accumulation des indices suggérant de reconnaittre dans les restes de l'édifice néronien mis au jour le soubassement de la cenatio rotunda du palais de Néron renforcent notablement les arguments réunis en faveur de cette identification ${ }^{2}$. En revanche, les informations disponibles laissent encore un large champ aux hypothèses touchant le fonctionnement du mécanisme et l'aspect de l'édifice.

2 Au-delà des considérations pouvant être faites sur l'interprétation de l'édifice, le caractère incontestablement exceptionnel de ce dernier impose une publication rapide des observations, ainsi que des hypothèses et des commentaires qu'il inspire. C'est dans cette perspective que se sont orientées toutes les interventions effectuées sur le terrain en 2015.

\section{Interventions sur le terrain}

3 L'obtention de la concession Prot. N. DG 5008, datée du 12/06/2015, accordée à l'École française de Rome par le Ministero per i Beni Culturali e il Turismo (MiBACT), a permis aux personnes qui étudient le mobilier d'accéder au dépôt installé sur le site de la Vigna Barberini. Divers travaux ont ainsi pu être réalisés, principalement par M. Fedeli, L. David, G. Rizzo et J. Tuosto. Cette dernière a traité les céramiques à paroi fine dans le cadre d'un mémoire de master 1 (Università Roma Tre), tandis que les trois autres effectuaient des contrôles, des compléments d'identification des céramiques, ainsi que les dessins des objets les plus remarquables. L'ensemble des données qu'ils ont ainsi réunies doit confluer dans la publication en cours de préparation. Il en ira de même pour l'étude de M. Carrive portant sur les restes de peintures antiques, celles de M. Rossi sur les terres cuites architecturales, de R. Montalbano sur les décors marmoréens et de V. Fontana sur les monnaies, qui ont dû tous les quatre reprendre en mains le mobilier en 2015, tandis 
que d'autres travaillaient sur les informations collectées antérieurement, principalement en 2014.

4 Il a également été possible d'accéder au site et, en juillet, l'architecte de l'Institut de recherche en architecture antique (IRAA), N. André (AMU-CNRS), a ainsi pu intervenir pour compléter les relevés des structures, avec la responsable de l'opération, ainsi que E. Battiante, une stagiaire de l'Université La Sapienza. En parallèle, Ch. Durand, photographe du Centre Camille Jullian (AMU-CNRS), se chargeait de compléter la couverture des vestiges et de photographier les objets importants, tandis que son collègue L. Damelet (AMU-CNRS) expérimentait un système de relevé photogrammétrique à l'intérieur de l'escalier hélicoïdal.

\section{Publication des fouilles de la cenatio rotunda}

Bien des aspects du bâtiment néronien restant encore très flous, on ne peut envisager de fournir qu'un bilan des éléments connus à ce jour, assorti d'hypothèses de reconstruction. Une des ambitions de cette publication sera de livrer une description précise et détaillée à tous ceux qui s'intéressent à la découverte, afin de susciter d'autres réflexions sur le sujet. Ce dernier ne sera certainement pas épuisé dans le cadre de ce volume, avant tout parce que, au stade actuel, les informations recueillies ne conduisent que rarement à des certitudes, et seule la reprise des fouilles pourrait aider à asseoir plus solidement les interprétations.

Considérant qu'il est nécessaire et utile de présenter la construction néronienne dans son contexte stratigraphique, en particulier en raison des conséquences que les épisodes postérieurs ont eues sur sa conservation et donc sur nos connaissances, il a été décidé de dresser un tableau couvrant toutes les périodes de l'histoire du site. Toutefois, sachant qu'une étude exhaustive de l'ensemble du mobilier et du bâti engendrerait des temps de préparation très longs, nous avons choisi d'adopter deux niveaux de lecture, en exposant d'abord toutes les observations faites en examinant la stratification, puis en approfondissant l'analyse consacrée aux vestiges néroniens, avant de replacer ceux-ci dans leur contexte historique, symbolique et culturel.

7 Au cours de l'année 2015, l'École française de Rome a organisé deux rencontres destinées à réunir la plupart des participants au projet, principalement des personnes qui sont intervenues sur le terrain, et/ou au stade de l'exploitation du mobilier, mais également quelques experts susceptibles d'élargir le point de vue des fouilleurs. Durant la première, qui a eu lieu en février, ont été discutés et définis les objectifs de la publication, ainsi que les contenus des contributions que les participants se sont engagés à fournir. En juin, la seconde réunion a permis de dresser un bilan des travaux en cours. Durant ces rencontres, plusieurs questions touchant l'interprétation de la structure, ainsi que celle de certains documents comme les monnaies de la série MAC AVG, ont fait l'objet de débats qui ont contribué à orienter les contenus de la publication.

\section{Plan du volume}

8 La première partie de l'ouvrage en cours de préparation est consacrée à l'exposition de l'ensemble des données livrées par la fouille. Elle compte deux chapitres, dont l'un se 
présente sous la forme d'une narration des événements dont le terrain a fourni des témoignages, tandis que le second regroupera des études portant sur le mobilier.

Afin de rendre plus fluide le compte rendu des épisodes de l'histoire du secteur, j'ai choisi de suivre l'ordre chronologique selon lequel ils se sont déroulés, en partant donc des quelques données disponibles sur les périodes précédant la construction de l'édifice néronien pour remonter progressivement jusqu'au $\mathrm{XX}^{\mathrm{e}}$ siècle. Dans le second chapitre interviennent d'abord L. David, M. Fedeli et G. Rizzo, qui ont élaboré des tableaux illustrant la composition du mobilier regroupé en fonction de l'attribution des strates à une période et à une phase de l'histoire du site. Les subdivisions feront écho à celles qui scandent le premier chapitre. Dans chaque cas, le premier tableau, très synthétique, est suivi par un tableau qui ne rassemble plus que les céramiques d'époque républicaine et impériale, classées en fonction de leur attribution à une production et à un type ou forme. Ces tableaux seront accompagnés d'un commentaire visant à définir le mode de formation des dépôts, ainsi que leur datation. S. Zeggio et F.R. Fiano soumettront ensuite les résultats de leur étude des céramiques antérieures au $\mathrm{IV}^{\mathrm{e}}$ siècle avant n.è., puis $\mathrm{M}$. Rossi ceux qui concernent les terres cuites architecturales. Suivront les présentations des enduits peints (M. Carrive), des monnaies (V. Fontana), des estampilles sur briques (F. Chausson), des revêtements de marbre (N. Delferrière) et de quelques fragments de décor architectural remarquables (R. Montalbano).

Dans la deuxième partie du volume, l'intérêt se concentre exclusivement sur l'édifice néronien, dont on analysera les rapports avec le relief, avant de décrire la construction, les matériaux employés et les caractéristiques de la réalisation (N. André et F. Villedieu). Le mécanisme et tous les aménagements qui semblent en rapport avec lui sont étudiés par P. Vitti et M. Gabay. Ceux-ci avanceront des hypothèses sur le fonctionnement de l'ensemble. Une contribution, signée par M. Carrive, réunit des œuvres peintes et sculptées sur lesquelles apparaissent des édifices de plan circulaire présentant des traits de parenté avec celui de la Vigna Barberini ; elle fournit des pistes qui seront exploitées par N. André et J.-M. Gassend pour proposer des reconstructions hypothétiques du bâtiment. Deux contributions de $\mathrm{M}$. A. Tomei ont pour thème l'une l'alimentation en eau de ce secteur du Palatin, tandis que l'autre rassemble les éléments connus pouvant avoir appartenu à la partie de la résidence de Néron située sur le Palatin. Enfin, E. Dumas et E. Battiante complèteront ce dernier travail en proposant une vue d'ensemble des vestiges attribués à la domus Transitoria et/ou à la domus Aurea sous la forme d'un plan accompagné de références et commentaires.

11 La troisième et dernière partie réunira des contributions traitant l'une de réalisations antérieures qui annoncent celle des ingénieurs de Néron (F.Coarelli); l'autre des significations symboliques et idéologiques du même édifice (P.Gros, Vice mundi); une troisième du rapport entre architecture et paysage (C. Cecamore, Lo spazio conquistato); dans la dernière, C. Devoto, F. Catalli et B. Spigola exposeront les résultats de l'enquête qu'ils ont menée sur les monnaies de la série MAC AVG et leurs réflexions sur le sujet.

12 L'ouvrage, qui devrait paraître dans la collection Roma Antica, sera introduit par G. Morganti, qui rappellera les circonstances de la découverte.

13 Fin 2015, la préparation du volume est bien avancée mais encore assez loin de sa conclusion dans la mesure où certaines études n'ont été qu'ébauchées et que la préparation des plans et autres relevés nécessitera encore plusieurs mois de travail. 


\section{Activités de valorisation}

14 L'équipe a été sollicitée à plusieurs reprises pour présenter les résultats des recherches dans le cadre de manifestations visant à offrir au grand public une image des recherches scientifiques en cours ${ }^{3}$, pour fournir des éléments à des journalistes travaillant pour la presse et pour la télévision ${ }^{4}$. N. André a été amenée pour cela à fournir des restitutions en 3D de l'édifice et plus particulièrement de l'escalier à vis.

\section{Projets}

15 Parmi les travaux que l'on envisage de mener durant l'année 2016, se trouve au premier rang la conclusion de la préparation du volume décrit ci-dessus.

Une demande de prorogation de la concession de fouille a été déposée auprès du MiBACT dans le but d'une part de poursuivre les recherches portant sur le mobilier, d'autre part de compléter la couverture photogrammétrique de l'escalier à vis. Dans le premier cas, l'objectif sera de préparer la publication du mobilier mis au jour dans des canaux du réseau d'assainissement du palais impérial flavien. Il s'agit là d'un lot extrêmement riche (15 366 fragments répertoriés), qui se forme durant les dernières années du II siècle et offre donc une image assez exhaustive des céramiques en usage à ce moment précis.

La reprise des relevés photogrammétriques aura pour but d'obtenir des images à très haute densité, présentant donc des qualités esthétiques, mais aussi l'avantage de permettre une étude fine de la construction en rendant possible le repérage de détails techniques, que les conditions de travail normales ne permettent pas d'effectuer. L'intervention présentera un caractère expérimental, dans la mesure où il faut élaborer des solutions techniques afin d'obtenir une illumination uniforme des structures et où le traitement des données devient difficile lorsque les images acquièrent une densité élevée. L'expérimentation sera réalisée par N. André, de l'IRAA, par L. Damelet, du CCJ, en collaboration avec l'auteur du logiciel CloudCompare. L'importance en temps et moyens de l'opération dépendra du succès éventuel de demandes de financement qui ont été déposées et dont on ne sait pas encore si elles seront retenues. À plus ou moins long terme, ces images seront utilisées pour réaliser une visite virtuelle de l'escalier.

Enfin, nous envisageons de préparer des propositions en réponse à des appels à projets européens, pour poursuivre les recherches et éventuellement reprendre les fouilles sur le terrain.

\section{BIBLIOGRAPHIE}

Coarelli 2012 = F. Coarelli, Palatium : il Palatino dalle origini all'Impero, Rome, 2012.

Villedieu 2010 = Fr. Villedieu, La cenatio rotunda de la Maison Dorée de Néron, dans CRAI, 2010, III, p. 1089-1114. 
Villedieu 2011a = Fr. Villedieu, Une construction néronienne mise au jour sur le site de la Vigna Barberini : la cenatio rotunda de la Domus Aurea ?, dans Neronia electronica, fasc. 1, 2011, p. 37-52 [En ligne] URL : http://www.sien-neron.fr/2011/11/neronia-electronica-\%E2\%80\%93fascicule-1-2011/.

Villedieu 2011b = Fr. Villedieu, $L a$ « Coenatio rotunda » neroniana e altre vestigia nel sito della Vigna Barberini al Palatino, dans Bollettino d'Arte, 12, 2011 (VI), p. 1-28.

Villedieu 2012 = Fr. Villedieu, « Palatin (Rome) », Chronique des activités archéologiques de l'École française de Rome [En ligne], mis en ligne le 19 décembre 2012, URL : http://cefr.revues.org/318.

Villedieu 2015 = Fr. Villedieu, « Vigna Barberini (Palatin, Rome) », Chronique des activités archéologiques de l'École française de Rome [En ligne], Italie centrale, mis en ligne le 16 février 2015. URL : http://cefr.revues.org/1323.

\section{NOTES}

1. Les résultats de ces campagnes ont été exposés principalement dans les articles suivants: Villedieu 2010 ; Villedieu 2011a ; Villedieu 2011b ; Villedieu 2012 ; Villedieu 2015.

2. Voir les articles cités note 1 , ainsi que Coarelli 2012, p. 504 principalement.

3. "L'archéologie à "Innovatives SHS 2015" » le 16 juin 2015, Centre des Congrès de la Villette, à la Cité des sciences et de l'industrie, Paris, stand D8 ; "Journées nationales de l'archéologie », 19-21 juin 2015, à Marseille ; "Fête de la Science-Le Village des sciences », Aix-en-Provence, 9-11 octobre 2015 ; conférence dans le cadre de la « Fête de la science 2015 » et des « Jeudis du CNRS », 8 octobre 2015, à Marseille.

4. Le journal Sciences et Vie Junior a publié un article intitulé Néron le mégalo, dans le n 307, avril 2015, p. 48-53, signé par Olivier Lapirot et Serge Lathière. Par ailleurs, une équipe est intervenue en juillet sur le terrain et en laboratoire pour préparer un documentaire de la série ARKEO, destiné à être présenté sur ARTE.

\section{INDEX}

Mots-clés : cenatio rotunda, domus aurea, palais, Palatin, Néron

\section{AUTEUR}

\section{FRANÇOISE VILLEDIEU}

Aix Marseille Université, Centre Camille Jullian (UMR 7299 du CNRS) - francoise.villedieu [at]gmail.com 